\title{
The Effect of Blood Loss on the Hemodynamics of Pediatric Patients Undergoing Simultaneous Bilateral Cochlear Implantation
}

\author{
Original Investigation $>$ Berat Demir $^{1}$ (D), Adem Binnetoğlu² (D), Çaglar Batman ${ }^{1}$ (D) \\ ${ }^{1}$ Department of Otorhinolaryngology-Head and Neck Surgery, Marmara University School of Medicine, Pendik Training and \\ Research Hospital, İstanbul, Turkey \\ ${ }^{2}$ Department of Otorhinolaryngology-Head and Neck Surgery, St. Elizabeth's Medical Center, Brighton, MA, USA
}

Abstract

ORCID iDs of the authors: B.D. 0000-0002-8574-4927; A.B. 0000-0002-2797-0248; Ç.B. 0000-0002-7807-6895.

Cite this article as: Demir B, Binnetoğlu A, Batman Ç. The Effect of Blood Loss on the Hemodynamics of Pediatric Patients Undergoing Simultaneous Bilateral Cochlear Implantation. Turk Arch Otorhinolaryngol 2020; 58(2): 118-21.

\section{Corresponding Author:}

Berat Demir, drberatdemir80@hotmail.com

Received Date: 17.01.2020

Accepted Date: 02.04 .2020

Content of this journal is licensed under a Creative Commons Attribution 4.0 International License. Available online at www.turkarchotolaryngol.net
Objective: Our objectives were to find the mean blood loss volume and the mean hemoglobin decrease in patients undergoing simultaneous bilateral cochlear implant surgery, to determine whether they had clinical effects, and to calculate a minimum weight for pediatric patients that is independent of any age criteria.

Methods: A retrospective chart review of the preand post-operative hemoglobin concentrations and mean blood loss volumes were calculated for children between 12-24 months of age who underwent cochlear implant surgery.

Results: A postoperative decline in hemoglobin concentration was found in 67 cases (92\%), with a mean difference between the pre- and post-operative values measuring $1.9 \mathrm{~g} / \mathrm{dL}$. Preoperative hemoglobin concentrations decreased from $13.4 \mathrm{~g} / \mathrm{dL}$ to $11.5 \mathrm{~g} / \mathrm{dL}$. Mean blood loss volume was $82.3 \pm 12 \mathrm{cc}$.

Conclusion: Although simultaneous bilateral cochlear implant surgery is reimbursed by the state for those who are greater than one year of age and who meet the appropriate audiological criteria, our findings suggest that these patients should weigh $\geq 10 \mathrm{~kg}$ to prevent hemodynamic instability due to blood loss during surgery.

Keywords: Child, cochlear implantation, hemodynamics, hemoglobin, ideal body weight

\section{Introduction}

Cochlear implantation (CI) has become a routine procedure for the management of profound sensorineural hearing loss (SNHL), and bilateral CI surgeries are performed worldwide (1). Compared to unilateral implantation, bilateral CI has several advantages such as enabling to determine the direction of the sound, making hearing more understandable, and reducing the head shadow effect (2). Along with the risks of CI surgery, there are risks associated with anesthesia, especially in the pediatric patient group. Advancements in clinical audiology and pediatric anesthesia have allowed for shorter times to CI surgery in younger children. However, both younger age and bilateral implantation may increase surgical and anesthetic risks. On the other hand, it is widely accepted that no significant bleeding occurs during CI surgery (3). Because of their anatomical and physiological characteristics, blood loss can easily disrupt hemodynamic stability in children; bleeding from the emissary vein may cause such disruption (3). Considering that the average weight of a 12-monthold child is approximately $10 \mathrm{~kg}$, the maximum blood loss that a child can tolerate is $80 \mathrm{cc}$ (3). This type of hemorrhage, which is thought not to be abundant in terms of bilateral CI surgery, may impair hemodynamic stability in the child. In accordance with the information provided in the study, we aimed to find the mean blood loss volume and mean decrease in hemoglobin concentration that occur during bilateral CI surgery. In the present study we aimed to determine the effect of blood loss volume on the hemodynamics of infants who underwent bilateral $\mathrm{CI}$ surgery by measuring their hemoglobin levels and hemoglobin concentrations.

\section{Methods}

Two-hundred-and-sixty-seven CI were performed in the Department of Otorhinolaryngology of Marmara University Hospital from January 
2017 through May 2019. During this period, we examined 73 patients who were aged between 12-24 months, weighed more than 10 kilograms, and underwent bilateral simultaneous CI (Table 1). A total of 194 patients were excluded from the study because of a history of unilateral surgery, a bilateral surgery that was not performed simultaneously, age greater than 24 months at the time of the CI, bleeding disorders, or weight less than 10 kilograms. The study was approved by the Ethics Committee of Marmara University with number 10.2019 .799 on May 08, 2020. Informed consent was obtained from the parents of the patients. This study was performed retrospectively to compare preoperative and postoperative hemoglobin concentrations and to investigate the clinical implications of this condition. Patient files, anesthesia charts, operative findings, and biochemical investigations were retrospectively reviewed. The circulating blood volume in children was calculated as $80 \mathrm{cc} / \mathrm{kg}$ (4).

All surgeries were performed by the same surgical team. A soft insertion technique was used in the surgeries. A lazy-S incision, mastoidectomy, posterior tympanotomy, an oval window or cochleostomy approach in general anesthesia were used in all $\mathrm{pa}^{-}$ tients to place the CI. Duration of surgery was recorded.

Preoperative hemogram, prothrombin time, and partial thromboplastin time were evaluated in all patients. Hemogram was measured in all patients on the first postoperative day. Patients with a decreased hemoglobin level $(<7 \mathrm{~g} / \mathrm{dL})$ and/or with symptoms of tachycardia and hypotension received blood transfusion.

Table 1. Demographics of patients

\begin{tabular}{l|c}
\hline Sex & $\mathbf{n}(\%)$ \\
\hline Boys & $39(53.4)$ \\
\hline Girls & $34(46.6)$ \\
\hline Age & (months) \\
\hline Mean \pm SD & $14.4 \pm 2.1$ \\
\hline Brand of cochlear implant & $\mathbf{n}$ \\
\hline Nucleus Contour Advance & 52 \\
\hline MED-EL medium & 16 \\
\hline Nucleus Straight & 5 \\
\hline n: number of patients, SD: standard deviation &
\end{tabular}

\section{Main Points}

- Blood loss can easily disrupt hemodynamic stability in children because of their anatomical and physiological characteristics.

- The total blood volume, and therefore the total weight of children is important in bilateral CI surgery, as blood loss causes hemodynamic instability and transfusion can be necessary of more than $10 \%$.

- Given the amount of hemorrhage and the possible reduction in hemoglobin concentration, patients should have an ideal weight of at least $10 \mathrm{~kg}$ before undergoing a bilateral CI procedure.

- Bilateral simultaneous CI surgery in those over the age of oneespecially in children approximately 12 months of age-should not only be based on audiological indications but also on the weight of the child.
Intraoperative bleeding volumes of the patients were retrieved from the anesthesia forms in the patient files; objective blood losses were estimated based on the volume of blood passing through the suction container (since washing liquid is used for reasons such as burring, this amount was deducted from the total) and the amount of blood absorbed by gauzes used during the operation. Balanced isotonic electrolyte solutions were used for fluid replacement in all patients. Intraoperative fluid management was performed according to the Holliday-Segar formula (4), and since the average duration of surgery was two hours, fluid was given to patients with an average of $60 \mathrm{cc} / \mathrm{kg}$.

\section{Statistical Analysis}

Data analyses were undertaken using Statistical Package for the Social Sciences software for Windows version 16.0 (SPSS Inc., Chicago, IL, USA).

\section{Results}

There were 39 (53.4\%) boys and 34 (46.6\%) girls in the study. Their mean age was 14.7 months $( \pm 2.1)$. Nucleus Freedom Contour ${ }^{\circledR}$ Advance (Cochlear Ltd., Lane Cove NSW, Australia) was implanted in 52, Med-El Medium (Medel Medical Electronics) in 16, and Nucleus CI422 Straight (Cochlear Ltd.) in five children (Table 1). A postoperative decline in hemoglobin concentration was found in 67 cases (92\%), with a mean difference between the pre- and post-operative values that measured -1.9 $\mathrm{g} / \mathrm{dL}$. Mean postoperative hemoglobin values decreased to 11.5 $\mathrm{g} / \mathrm{dL}$ from preoperative $13.4 \mathrm{~g} / \mathrm{dL}$. Mean blood loss volume was found to be $82.3 \pm 12 \mathrm{cc}$. With the exception of one patient, no additional treatment was required due to stable clinical course. Blood transfusion was necessary for one patient who had a hemoglobin level $<7 \mathrm{~g} / \mathrm{dL}$ and hemodynamic instability. No additional plasma, platelet, fibrinogen or tranexamic acid transfusion was required in any of the patients. Bleeding occurred primarily from the emissary veins while separating the muscle-periosteal flap from the mastoid bone. Surgical duration was $124.7 \pm 11.9$ min. No hematoma was seen. No surgical complication was observed in any of the patients.

\section{Discussion}

CI surgery has evolved through various techniques that have been developed to reduce the occurrence of postoperative complications. Although CI surgery has been successfully performed in many patients, surgery related complications continue to occur. Potential complications associated with CI in children include facial nerve injury (paralysis or paresis), eardrum perforation, vertigo, device failure, device extrusion, electrode migration, trauma to the implant site, damage to the receiver-stimulator, acute infection of the middle ear and the mastoid, or development of a cholesteatoma (5). These complications are classified as being major or minor (6). Bleeding is classified as a minor complication, even though it can cause significant hemorrhage or hematoma. However, it was seen as a serious loss with a mean hemoglobin concentration decrease measuring $1.9 \mathrm{mg} / \mathrm{dL}$ in our pediatric patients. Contrary to popular belief, this is evidence that CI surgery is not a bloodless procedure. The total blood volume, and therefore the to- 
tal weight, of children is important in bilateral CI surgery, as blood loss causes hemodynamic instability and transfusion can be necessary of more than $10 \%$. The mean volume of blood loss was determined to be $82.3 \mathrm{cc}$; considering the $10 \%$ ratio, this estimates the total blood volume to be 750-800 cc, which corresponds to a body weight of at least $10 \mathrm{~kg}$. Hemodynamic instability in children can be expected when bleeding is over $10 \%$ (7). This corresponds to approximately $80 \mathrm{cc}$ of bleeding. Although James and Papsin (8) reported that a unilateral CI with careful surgery would not result in $80 \mathrm{cc}$ of bleeding, this amount of bleeding is more likely to occur when bilateral simultaneous CI surgery is performed.

The mean difference between pre- and post-operative hemoglobin concentrations was $1.9 \mathrm{~g} / \mathrm{dl}$. While this is an important decrease in pediatric patients, only one child in our study group required transfusion. Anagiotos and Beutner (3) reported that banked human blood should be obtained before performing a bilateral simultaneous CI surgery. Although this decrease in hemoglobin value is used to calculate the transfusion volume (9), there is no certain hemoglobin concentration accepted for transfusion volume (10). In contrast, the formula that determines the required volume of transfusion is based on the current and the desired hemoglobin levels (9). A hemoglobin level of less than $6 \mathrm{mg} / \mathrm{dL}$ is the lowest recommended value for transfusion (10). Transfusion certainly is not determined based only on hemoglobin values and blood loss. Blood pressure, heart rate, oxygen saturation, electrocardiography, echocardiography, urine output, cerebral monitoring (i.e., cerebral oximetry), analysis of arterial blood gasses, and mixed venous oxygen saturation are other parameters related to transfusion requirement (11).

Our findings showed that most of the bleeding was observed in the emissary veins. Bipolar and/or monopolar cautery was used in CI surgery; however, it is not easy to stop bleeding from the emissary veins. In our cases, we found that hemostats such as bone wax reduced the amount of bleeding. In addition, a diamond burr was used to shave the bone marrow to control and reduce bleeding. Furthermore, we do not routinely prepare blood for any patient before surgery because the preoperative weight of the selected patients is $\geq 10 \mathrm{~kg}$. Another important reason is that children do not have additional diseases. For those with another disease, such as preoperative anemia or cyanotic heart disease, a certain hemoglobin value was not measured and preoperative blood provision was recommended (10). Besides, blood transfusions should be considered in cases of severe hypoxemia, active blood loss, or hemodynamic instability (10). An experienced anesthesiologist and an experienced pediatrician should therefore be part of the CI team.

There are studies in the literature concerning the amount of bleeding that occurs in children under one year of age who undergo CI surgery $(8,11,12)$. In Turkey, however, the state reimbursement plan covers those over one year of age, hence we do not have any experience with bleeding in those under one year of age on official records. Therefore, this is the first Turkish study to examine bleeding in those undergoing bilateral simultaneous
CI surgery. Future studies should be conducted with the participation of more patients and centers, and their findings should be discussed.

\section{Conclusion}

With the spread of bilateral simultaneous CI surgery, problems of intraoperative blood loss have been raised in addition to the other complications of the procedure. Mild bleeding can affect the hemodynamics in the pediatric patient group-especially in those approximately 12 months of age. Therefore, in Turkey, bilateral simultaneous CI surgery in those over the age of oneespecially in children approximately 12 months of age- should not only be based on audiological indications but also on the weight of the child. Given the amount of hemorrhage and the possible reduction in hemoglobin concentration, we recommend that patients should have an ideal weight of at least $10 \mathrm{~kg}$ before undergoing a bilateral $\mathrm{CI}$ procedure.

Ethics Committee Approval: Ethics committee approval was received for this study from the Ethics Committee of Marmara University (Approval Date: May 8, 2020; Approval Number: 10.2019.799).

Informed Consent: Informed consent was obtained from the parents of the patients who participated in this study.

Peer-review: Externally peer-reviewed.

Author Contributions: Concept - B.D.; Design - A.B.; Materials B.D.; Data Collection and/or Processing - B.D.; Analysis and/or Interpretation - A.B.; Literature Search - A.B.; Writing - A.B.; Critical Reviews - Ç.B.

Conflict of Interest: The authors have no conflicts of interest to declare.

Financial Disclosure: The authors declared that this study has received no financial support.

\section{References}

1. Demir B, Binnetoglu A, Mammodova U, Batman C. Scar evaluation in subperiosteal temporal pocket versus the one-layer flap technique in cochlear implantation using the patient and observer scar assessment scale. Eur Arch Otorhinolaryngol 2019; 276: 2149-54. [Crossref]

2. Migirov L, Kronenberg J. Bilateral, simultaneous cochlear implantation in children: surgical considerations. J Laryngol Otol 2009; 123: 837-9. [Crossref]

3. Anagiotos A, Beutner D. The impact of blood loss during cochlear implantation in very young children. Eur Arch Otorhinolaryngol 2013; 270: 2439-44. [Crossref]

4. Holliday MA, Segar WE. The maintenance need for water in parenteral fluid therapy. Pediatrics 1957; 19: 823-32.

5. Chiesa Estomba CM, Rivera Schmitz T, Betances Reinoso FA, Dominguez Collado L, Estevez Garcia M, Lorenzo AI. Complications after cochlear implantation in adult patients. 10-year retrospective analysis of a tertiary academic centre. Auris Nasus Larynx 2017; 44: 40-5. [Crossref]

6. Park C, Wormald J, Miranda BH, Ong J, Hare A, Eccles S. Perioperative blood loss and transfusion in craniosynostosis surgery. J Craniofac Surg 2018; 29: 112-5. [Crossref] 
7. Battersby EF. Paediatric anaesthesia. In: Adams DA, Cinnamond MJ (eds) Scott-Brown's otolaryngology. Butterworth-Heinemann, Oxford; 1997.p.6-24.

8. James AL, Papsin BC. Cochlear implant surgery at 12 months of age or younger. Laryngoscope 2004; 114: 2191-5. [Crossref]

9. Howie SR. Blood sample volumes in child health research: review of safe limits. Bull World Health Organ 2011; 89: 46-53. [Crossref]

10. Ali N. Red blood cell transfusion in infants and children - current perspectives. Pediatr Neonatol 2018; 59: 227-30. [Crossref]
11. American Society of Anesthesiologists Task Force on Perioperative Blood Management. Practice guidelines for perioperative blood management: an updated report by the American Society of Anesthesiologists Task Force on Perioperative Blood Management. Anesthesiology 2015; 122: 241-75. [Crossref]

12. Cosetti M, Roland JT Jr. Cochlear implantation in the very young child: issues unique to the under-1 population. Trends Amplif 2010; 14: 46-57. [Crossref] 